\title{
RESTRICTION ESTIMATES VIA THE DERIVATIVES OF THE HEAT SEMIGROUP AND CONNECTION WITH DISPERSIVE ESTIMATES
}

\author{
Frédéric Bernicot and El MaAti Ouhabaz
}

\begin{abstract}
We consider an abstract non-negative self-adjoint operator $H$ on an $L^{2}$ space. We derive a characterization for the restriction estimate $\left\|\frac{d E_{H}(\lambda)}{d \lambda}\right\|_{L^{p} \rightarrow L^{p^{\prime}}} \leq$ $C \lambda^{\frac{d}{2}\left(\frac{1}{p}-\frac{1}{p^{\prime}}\right)-1}$ (involving the Radon-Nikodym derivative of the spectral measure) in terms of higher order derivatives of the semigroup $e^{-t H}$. We provide an alternative proof of a result in [1] which asserts that dispersive estimates imply restriction estimates. We also prove $L^{p}-L^{p^{\prime}}$ estimates for the derivatives of the spectral resolution of $H$.
\end{abstract}

\section{Introduction and main results}

Let $(X, \mu)$ be a measured space. That is $X$ is a non-empty set endowed with a positive measure $\mu$. We consider a non-negative self-adjoint operator $H$ on $L^{2}=L^{2}(X, \mu)$. We denote by $d E_{H}$ the spectral resolution of $H$ and by $\frac{d E_{H}}{d \lambda}$ its Radon-Nikodym derivative. Since we will be interested in $L^{p}-L^{p^{\prime}}$ estimates for $\frac{d E_{H}(\lambda)}{d \lambda}$ we shall assume throughout this note that the spectrum of $H$ is continuous. The $L^{p}-L^{p^{\prime}}$ norm will be denoted by $\left\|\frac{d E_{H}(\lambda)}{d \lambda}\right\|_{L^{p} \rightarrow L^{p^{\prime}}}$ and $p^{\prime}$ is the conjugate number of $p$.

We first discuss the Euclidean Laplacian. Suppose that $X=\mathbb{R}^{d}$ and $H=-\Delta$ (the positive Laplace operator) on $L^{2}\left(\mathbb{R}^{d}\right)$. It is a well-known fact that as a consequence of the Stein-Tomas estimates for the restricted Fourier transform to the unit sphere, the derivative of spectral measure $\frac{d E_{-\Delta}(\lambda)}{d \lambda}$ is a bounded operator from $L^{p}$ into $L^{p^{\prime}}$ for all $p \leq \frac{2 d+2}{d+3}$. In addition,

$$
\left\|\frac{d E_{-\Delta}(\lambda)}{d \lambda}\right\|_{L^{p} \rightarrow L^{p^{\prime}}} \leq C \lambda^{\frac{d}{2}\left(\frac{1}{p}-\frac{1}{p^{\prime}}\right)-1}, \quad \lambda>0 .
$$

Such estimate is sometimes referred to as the $(p, 2)$ restriction estimate of SteinTomas. We refer to the introductions of the papers $[1,3]$ for more details about this.

The above restriction estimate was extended to the setting of asymptotically conic manifolds in [3]. In the paper [1], the restriction estimate

$$
\left\|\frac{d E_{H}(\lambda)}{d \lambda}\right\|_{L^{p} \rightarrow L^{p^{\prime}}} \leq C \lambda^{\frac{d}{2}\left(\frac{1}{p}-\frac{1}{p^{\prime}}\right)-1},
$$

Received by the editors April 11, 2013.

2000 Mathematics Subject Classification. 47D03 ; 35P05 ; $42 \mathrm{~B} 15$.

Keywords and phrases. Restriction estimates; semigroup; spectral multipliers; dispersive estimates. 
was studied in an abstract setting. (Here $d$ is any positive constant). It is also proved there that (1.1) holds for several operators.

One of the aims of this note is to prove other characterizations of (1.1) in an abstract setting. In the following result we show that the restriction estimate for $H$ can be characterized in terms of higher order derivatives of the corresponding semigroup $e^{-t H}$. More precisely,

Theorem 1.1. Let $d$ be a positive constant and fix $p \in[1,2)$. The following assertions are equivalent.

(1) The restriction estimate (1.1) holds for every $\lambda>0$;

(2) There exists a positive constant $C$ such that

$$
\left\|H^{N} e^{-t H}\right\|_{L^{p} \rightarrow L^{p^{\prime}}} \leq C(N-1) ! N^{\frac{d}{2}\left(\frac{1}{p}-\frac{1}{p^{\prime}}\right)} t^{-N-\frac{d}{2}\left(\frac{1}{p}-\frac{1}{p^{\prime}}\right)},
$$

for all $t>0$ and all $N \in \mathbb{N}$;

(3) There exists a positive constant $C$ such that

$$
\|F(H)\|_{L^{p} \rightarrow L^{p^{\prime}}} \leq C R^{\frac{d}{2}\left(\frac{1}{p}-\frac{1}{p^{\prime}}\right)} \int_{\mathbb{R}}|F(s)| \frac{d s}{s},
$$

for all $R>0$ and bounded measurable function $F$ with supported in $[0, R]$.

The main novelty here is the characterization of (1.1) by (1.2). The equivalence of (1.1) and (1.3) is in the spirit of Proposition 2.4, Section 2.2 in [1]. Note however that in contrast to that proposition in [1] we do not assume here that the volume of balls in $X$ is polynomial. Moreover, the $L^{1}$ norm in the RHS in (1.3) is taken w.r.t. $\frac{d s}{s}$ rather than $d s$ as in [1] and it is obvious that

$$
\int_{0}^{R}|F(s)| \frac{d s}{s}=\int_{0}^{1}|F(R s)| \frac{d s}{s} \geq \int_{0}^{1}|F(R s)| d s .
$$

One of the main ingredients in the proof of Theorem 1.1 is the following result, which expresses the spectral measure in terms of the semigroup. We denote by $\langle.,$. the scalar product of $L^{2}$. We have

Theorem 1.2. Consider a bounded and continuous function $\phi$. Then for every $f, g \in$ $L^{2}$ we have

$$
\lim _{N \rightarrow \infty} \frac{1}{(N-1) !} \int_{0}^{\infty} \phi\left(s^{-1}\right)\left\langle((N-1) s H)^{N} e^{-s(N-1) H} f, g\right\rangle \frac{d s}{s}=\langle\phi(H) f, g\rangle .
$$

A useful consequence of the latter theorem is the following equality for the derivatives of $E_{H}(\lambda)$ (in which the limit has to be understood in the weak sense): for $k \geq 0$ an integer

$$
\frac{d^{k+1}}{d \lambda^{k+1}} E_{H}(\lambda)=\lim _{N \rightarrow \infty} \frac{1}{N !} \frac{d^{k}}{d \lambda^{k}}\left[\lambda^{-1}\left(N \lambda^{-1} H\right)^{N+1} e^{-N \lambda^{-1} H}\right]
$$

for $\lambda>0$. 
As an application we show that dispersive estimates for $H$ imply the restriction estimate (1.1) as well as $L^{p}-L^{p^{\prime}}$ estimates for the derivatives $\frac{d^{k+1}}{d \lambda^{k+1}} E_{H}(\lambda)$ for $k \leq$ $d / 2-1$. The estimates for the derivatives is new, whereas the case $k=0$ was already proved in [1].

We finish this introduction by explaining why it is interesting to prove the restriction estimate (1.1). Let us assume now that $(X, \mu)$ is equipped with a metric $\rho$ and assume that for every $x \in X, r>0$, the volume $\mu(B(x, r))$ of the open ball $B(x, r)$ satisfies

$$
c_{1} r^{d} \leq \mu(B(x, r)) \leq c_{2} r^{d}
$$

where $c_{1}$ and $c_{2}$ are positive constants. Suppose in addition that $H$ satisfies the finite speed of propagation property, that is the support of the kernel of $\cos (t \sqrt{H})$ is contained in $\{(x, y) \in X \times X, \rho(x, y) \leq t\}$. Under these assumptions, it is proved in [1] (see also [3] for the first assertion) that the restriction estimate implies sharp spectral multiplier theorems. More precisely,

Theorem 1.3. Suppose that the restriction estimate (1.1) holds for some fixed $p \in$ $[1,2)$. Then the following assertions hold.

(i) Compactly supported multipliers: Let $F$ be an even function with support in $[-1,1]$ and $F \in W^{\beta, 2}(\mathbb{R})$ for some $\beta>d(1 / p-1 / 2)$. Then $F(H)$ is bounded on $L^{p}(X)$, and

$$
\sup _{t>0}\|F(t H)\|_{L^{p} \rightarrow L^{p}} \leq C\|F\|_{W^{\beta, 2}} .
$$

(ii) General multipliers: Suppose that $F$ is an even bounded Borel function which satisfies $\sup _{t>0}\|\eta(\cdot) F(t \cdot)\|_{W^{\beta, 2}}<\infty$ for some $\beta>\max \{d(1 / p-1 / 2)$, $1 / 2\}$ and some non-trivial function $\eta \in C_{c}^{\infty}(0, \infty)$. Then $F(H)$ is bounded on $L^{r}(X)$ for all $p<r<p^{\prime}$. In addition,

$$
\|F(H)\|_{L^{r} \rightarrow L^{r}} \leq C_{\beta}\left(\sup _{t>0}\|\eta(\cdot) F(t \cdot)\|_{W^{\beta, 2}}+|F(0)|\right) .
$$

A version of this theorem for general doubling spaces is proved in [1]. One can apply Theorem 1.3 to prove summability results for Bochner-Riesz means on $L^{p}$-spaces.

\section{Proofs}

We start with the proof of Theorem 1.2. We shall write $\lesssim$ for $\leq$ up to a non-relevant constant $C$.

Proof of Theorem 1.2. Let us set $c_{N}^{-1}:=\int_{0}^{\infty} x^{N} e^{-x} \frac{d x}{x}=(N-1)$ ! By polarization, it suffices to prove that for every function $f \in L^{2}$

$$
\lim _{N \rightarrow \infty} c_{N} \int_{0}^{\infty} \phi\left(s^{-1}\right)\left\langle((N-1) s H)^{N} e^{-s(N-1) H} f, f\right\rangle \frac{d s}{s}=\langle\phi(H) f, f\rangle .
$$


We have

$$
\begin{aligned}
& c_{N} \int_{0}^{\infty} \phi\left(s^{-1}\right)\left\langle((N-1) s H)^{N} e^{-s(N-1) H} f, f\right\rangle \frac{d s}{s} \\
& =c_{N} \int_{0}^{\infty} \int_{0}^{\infty} \phi\left(s^{-1}\right)((N-1) s \lambda)^{N} e^{-(N-1) s \lambda}\left\langle d E_{H}(\lambda) f, f\right\rangle \frac{d s}{s} \\
& =\int_{0}^{\infty} \mu_{N}(\lambda)\left\langle d E_{H}(\lambda) f, f\right\rangle,
\end{aligned}
$$

where

$$
\mu_{N}(\lambda):=c_{N} \int_{0}^{\infty} \phi\left(s^{-1}\right)((N-1) s \lambda)^{N} e^{-(N-1) s \lambda} \frac{d s}{s} .
$$

Owing to the constant $c_{N}$, it is clear that the continuous function $\mu_{N}$ is bounded by $\|\phi\|_{L^{\infty}}$. Therefore, it is enough to prove that $\mu_{N}(\lambda)$ converges to the function $\phi(\lambda)$ for all $\lambda>0$ and then conclude by the dominated convergence theorem.

Taking the difference yields

$$
\begin{aligned}
\left|\mu_{N}(\lambda)-\phi(\lambda)\right| & =c_{N}\left|\int_{0}^{\infty}\left[\phi\left(s^{-1}\right)-\phi(\lambda)\right]((N-1) s \lambda)^{N} e^{-(N-1) s \lambda} \frac{d s}{s}\right| \\
& \leq \int_{0}^{\infty}\left|\phi\left(s^{-1}\right)-\phi(\lambda)\right| c_{N}((N-1) s \lambda)^{N} e^{-(N-1) s \lambda} \frac{d s}{s} .
\end{aligned}
$$

Using Stirling's formula

$$
c_{N}=[(N-1) !]^{-1} \simeq\left(\frac{e}{N-1}\right)^{N-1}(2 \pi N)^{-\frac{1}{2}},
$$

we obtain for large enough $N$ and uniformly with respect to $\lambda$

$$
\begin{aligned}
\left|\mu_{N}(\lambda)-\phi(\lambda)\right| & \lesssim N^{\frac{1}{2}} \int_{0}^{\infty}\left|\phi\left(s^{-1}\right)-\phi(\lambda)\right|(s \lambda)^{N} e^{-(N-1)(s \lambda-1)} \frac{d s}{s} \\
& \lesssim N^{\frac{1}{2}} \int_{0}^{\infty}\left|\phi\left(s^{-1}\right)-\phi(\lambda)\right|\left(s \lambda e^{-(s \lambda-1)}\right)^{N-1} \lambda d s .
\end{aligned}
$$

Using the continuity of $\phi$ at $\lambda$, we know that for every $\epsilon>0$, there exists $\delta$ such that for every $s>0$

$$
|s \lambda-1| \leq \delta \Longrightarrow\left|\phi\left(s^{-1}\right)-\phi(\lambda)\right| \leq \epsilon .
$$

So, we decompose the integral for $s \lambda \leq 1-\delta, 1-\delta \leq s \lambda<1+\delta$ and $s \lambda \geq 1+\delta$, obtaining three terms I, II and III. For the first term, we have (since $x \rightarrow x e^{-(x-1)}$ is non-decreasing for $x \in(0,1))$

$$
\begin{aligned}
\mathrm{I} & \lesssim N^{\frac{1}{2}}\left((1-\delta) e^{\delta}\right)^{N-1} \lambda\|\phi\|_{L^{\infty}}\left(\int_{s \lambda \leq 1-\delta} d s\right) \\
& \lesssim N^{\frac{1}{2}}\left((1-\delta) e^{\delta}\right)^{N-1}\|\phi\|_{L^{\infty}} .
\end{aligned}
$$

For the third term; we similarly have (since $x \rightarrow x e^{-(x-1)}$ is decreasing for $x \in(1, \infty)$ )

$$
\begin{aligned}
\mathrm{III} & \lesssim N^{\frac{1}{2}}\left((1+\delta) e^{-\delta}\right)^{N-2}\left(\int_{s \lambda \geq 1+\delta}(s \lambda) e^{-(s \lambda-1)} \lambda d s\right)\|\phi\|_{L^{\infty}} \\
& \lesssim N^{\frac{1}{2}}\left((1+\delta) e^{-\delta}\right)^{N-2}\|\phi\|_{L^{\infty}} .
\end{aligned}
$$


Because the map $x \mapsto x e^{1-x}$ attains its maximum (which is equal to 1 ) at $x=1$, then $(1-\delta) e^{\delta}<1$ and $(1+\delta) e^{-\delta}<1$ and so the two previous terms tend to 0 when $N$ goes to infinity. About the second term, we have the trivial bound

$$
\begin{aligned}
\mathrm{II} & \leq \int_{|s \lambda-1| \leq \delta} \epsilon c_{N}((N-1) s \lambda)^{N} e^{-(N-1) s \lambda} \frac{d s}{s} \\
& \leq \epsilon c_{N} \int_{0}^{\infty}((N-1) s \lambda)^{N} e^{-(N-1) s \lambda} \frac{d s}{s} \\
& \leq \epsilon .
\end{aligned}
$$

As a consequence, we deduce that for every $\epsilon>0$

$$
\limsup _{N \rightarrow \infty}\left|\mu_{N}(\lambda)-\phi(\lambda)\right| \leq \epsilon
$$

which concludes to the fact that $\mu_{N}$ pointwisely converges to $\phi$. Then, using dominated convergence Theorem and then spectral theory in (2.3) implies (2.1).

Remark 2.1. Let us point out that the previous reasoning does not allow us to describe the speed of convergence for $\mu_{N}$ to $\phi$. We want to detail that if we know the modulus of continuity of the function $\phi$ then we can have some information about the convergence. For example, let us assume that the function $\phi$ is $\rho$-Hölder at $\lambda$ for some $\rho \in(0,1)$.

In this case, as previously done we then decompose the integral in (2.4) for $s \lambda \leq u_{N}$, $u_{N}<s \lambda<v_{N}$ and $s \lambda \geq v_{N}$, obtaining three terms I, II and III and where $u_{N}<1$ and $v_{N}>1$ will be suitably chosen later (around 1). Then we have using the same arguments as before

$$
\mathrm{I} \lesssim N^{\frac{1}{2}}\left(u_{N} e^{-\left(u_{N}-1\right)}\right)^{N-1}\|\phi\|_{L^{\infty}}
$$

and

$$
\mathrm{III} \lesssim N^{\frac{1}{2}}\left(v_{N} e^{-\left(v_{N}-1\right)}\right)^{N-2}\|\phi\|_{L^{\infty}}
$$

About the second term, using the $\rho$-Hölder regularity of $\phi$ (and that $x e^{-(x-1)} \leq 1$ for every $x>0$ ), it follows that

$$
\begin{aligned}
\mathrm{II} & \lesssim N^{\frac{1}{2}} \int_{u_{N} \leq s \lambda \leq v_{N}}\left(\frac{v_{N}-u_{N}}{s}\right)^{\rho} \lambda d s \\
& \lesssim N^{\frac{1}{2}}\left(2 \lambda\left(v_{N}-u_{N}\right)\right)^{\rho}\left|v_{N}-u_{N}\right|
\end{aligned}
$$

where we used that $u_{N}, v_{N}$ are around the value 1 . Finally, we deduce that for every $\lambda$

$$
\begin{aligned}
\left|\mu_{N}(\lambda)-\phi(\lambda)\right| \lesssim & N^{\frac{1}{2}}\left(u_{N} e^{-\left(u_{N}-1\right)}\right)^{N-1} \\
& +N^{\frac{1}{2}}\left(v_{N} e^{-\left(v_{N}-1\right)}\right)^{N-2}+N^{\frac{1}{2}}\left(\lambda\left(v_{N}-u_{N}\right)\right)^{\rho}\left|v_{N}-u_{N}\right| .
\end{aligned}
$$

Now, let us write

$$
u_{N}:=1-\epsilon_{N} \quad \text { and } \quad v_{N}:=1+\epsilon_{N},
$$


with $\epsilon_{N} \rightarrow 0$ as $N \rightarrow \infty$. We note that by a second order expansion,

$$
\begin{aligned}
\log \left(N^{\frac{1}{2}}\left(u_{N} e^{-\left(u_{N}-1\right)}\right)^{N-1}\right) & =\frac{1}{2} \log (N)+(N-1)\left[\log \left(u_{N}\right)-\left(u_{N}-1\right)\right] \\
& =\frac{1}{2} \log (N)-(N-1)\left[\frac{1}{2}\left(u_{N}-1\right)^{2}+\mathcal{O}\left(u_{N}-1\right)^{3}\right] \\
& =\frac{1}{2} \log (N)-(N-1)\left[\frac{1}{2} \epsilon_{N}^{2}+\mathcal{O}\left(\epsilon_{N}^{3}\right)\right] \\
& \stackrel{N \rightarrow \infty}{\longrightarrow}-\infty
\end{aligned}
$$

provided

$$
\lim _{N \rightarrow \infty} \frac{N}{\log (N)} \epsilon_{N}^{2}=\infty
$$

In this case,

$$
\lim _{N \rightarrow \infty} N^{\frac{1}{2}}\left(u_{N} e^{-\left(u_{N}-1\right)}\right)^{N-1}=0
$$

Similarly, we have

$$
\lim _{N \rightarrow \infty} N^{\frac{1}{2}}\left(v_{N} e^{-\left(v_{N}-1\right)}\right)^{N-2}=0,
$$

and moreover, we can choose $\epsilon_{N}$ such that

$$
\lim _{N \rightarrow \infty} N^{\frac{1}{2}}\left|v_{N}-u_{N}\right|\left(2 \lambda\left(v_{N}-u_{N}\right)\right)^{\rho} \lesssim \lim _{N \rightarrow \infty} N^{\frac{1}{2}} \epsilon_{N}\left(\lambda \epsilon_{N}\right)^{\rho}=0 .
$$

Indeed, take $\epsilon_{N}$ such that

$$
N \epsilon_{N}^{2}=\log (N)^{2}
$$

(which is possible for large enough integer $N$ ) then $\epsilon_{N}$ tends to 0 and (2.5) and (2.6) are satisfied. For this choice, we let the reader to check that we have

$$
\left|\mu_{N}(\lambda)-\phi(\lambda)\right| \lesssim N^{-\sigma}
$$

for every $\sigma<\rho / 2$.

As a consequence, we deduce for example that for a smooth function $\phi$ compactly supported in $(0, \infty)$ then

$$
\left\|\frac{1}{(N-1) !} \int_{0}^{\infty} \phi\left(s^{-1}\right)((N-1) s H)^{N} e^{-s(N-1) H} \frac{d s}{s}-\phi(H)\right\|_{L^{2} \rightarrow L^{2}} \lesssim N^{-\sigma}
$$

for every exponent $\sigma<\frac{1}{2}$.

Corollary 2.2. Let $\delta \in(0,1]$. For every smooth function $\phi$ and every $L^{2}$-functions $f, g$, we have

$$
\begin{aligned}
\langle\phi(H) f, g\rangle & =\int_{0}^{\infty} \phi(\lambda)\left\langle\frac{d E_{H}(\lambda)}{d \lambda} f, g\right\rangle d \lambda \\
& =\lim _{N \rightarrow \infty} \frac{1}{(N-1) !} \int_{0}^{\infty} \phi(\lambda)\left\langle\left((N-\delta) \lambda^{-1} H\right)^{N} e^{-\lambda^{-1}(N-\delta) H} f, g\right\rangle \frac{d \lambda}{\lambda} \\
& =\lim _{N \rightarrow \infty} \frac{1}{\Gamma(N+\delta)} \int_{0}^{\infty} \phi(\lambda)\left\langle\left(N \lambda^{-1} H\right)^{N+\delta} e^{-\lambda^{-1} N H} f, g\right\rangle \frac{d \lambda}{\lambda}
\end{aligned}
$$


Proof. The case $\delta=1$ is exactly the statement of Theorem 1.2. For $\delta \in(0,1)$, we follow the same proof and replace $(N-1) !=\Gamma(N)$ by $\Gamma(N-\delta+1)$ (we recall that Stirling's formula remains valid for the $\Gamma$ function, see (2.7) below).

Making an integration by parts in Theorem 1.2, we obtain a formula for the derivatives $\frac{d^{k+1}}{d \lambda^{k+1}} E_{H}(\lambda)$ in terms of the semigroup. That is

Corollary 2.3. The following equality holds in the weak sense: for an integer $k \geq 1$

$$
\frac{d^{k+1}}{d \lambda^{k+1}} E_{H}(\lambda)=\lim _{N} \frac{1}{N !} \frac{d^{k}}{d \lambda^{k}}\left[\lambda^{-1}\left(N \lambda^{-1} H\right)^{N+1} e^{-N \lambda^{-1} H}\right]
$$

for $\lambda>0$.

Proof of Theorem 1.1. We first prove that (1) implies (2). Suppose that (1.1) is satisfied. For fixed $N$ we have

$$
\begin{aligned}
\left\|H^{N} e^{-t H}\right\|_{L^{p} \rightarrow L^{p^{\prime}}} & =\left\|\int_{0}^{\infty} \lambda^{N} e^{-t \lambda} d E_{H}(\lambda)\right\|_{L^{p} \rightarrow L^{p^{\prime}}} \\
& \lesssim \int_{0}^{\infty} \lambda^{N} e^{-t \lambda} \lambda^{\frac{d}{2}\left(\frac{1}{p}-\frac{1}{p^{\prime}}\right)-1} d \lambda \\
& =\left(\int_{0}^{\infty} u^{N+\frac{d}{2}\left(\frac{1}{p}-\frac{1}{p^{\prime}}\right)-1} e^{-u} d u\right) t^{-N-\frac{d}{2}\left(\frac{1}{p}-\frac{1}{p^{\prime}}\right)} . \\
& =t^{-N-\frac{d}{2}\left(\frac{1}{p}-\frac{1}{p^{\prime}}\right)} \Gamma\left(N+\frac{d}{2}\left(\frac{1}{p}-\frac{1}{p^{\prime}}\right)\right) .
\end{aligned}
$$

Stirling's formula for the Gamma function (see [2, Appendix A.6])

$$
\Gamma(x) \simeq x^{x-\frac{1}{2}} e^{-x} \sqrt{2 \pi} \text { for } x>0,
$$

shows that

$$
\left\|H^{N} e^{-t H}\right\|_{L^{p} \rightarrow L^{p^{\prime}}} \lesssim(N-1) ! N^{\frac{d}{2}\left(\frac{1}{p}-\frac{1}{p^{\prime}}\right)} t^{-N-\frac{d}{2}\left(\frac{1}{p}-\frac{1}{p^{\prime}}\right)} .
$$

This proves assertion (2).

We now prove that (2) implies (3). Suppose first that $F$ is a continuous function with support in $[0, R]$. We apply Theorem 1.2 and obtain

$$
\begin{aligned}
\|F(H)\|_{L^{p} \rightarrow L^{p^{\prime}}} \\
\quad \leq \limsup _{N} \frac{1}{(N-1) !} \int_{1 / R}^{\infty} F\left(s^{-1}\right)\left\|((N-1) s H)^{N} e^{-s(N-1) H}\right\|_{p-p^{\prime}} \frac{d s}{s} \\
\quad \lesssim \limsup _{N} \int_{1 / R}^{\infty} F\left(s^{-1}\right)((N-1) s)^{N} N^{\frac{d}{2}\left(\frac{1}{p}-\frac{1}{p^{\prime}}\right)}(s(N-1))^{-N-\frac{d}{2}\left(\frac{1}{p}-\frac{1}{p^{\prime}}\right)} \frac{d s}{s} \\
\quad \lesssim \int_{1 / R}^{\infty} F\left(s^{-1}\right) s^{-\frac{d}{2}\left(\frac{1}{p}-\frac{1}{p^{\prime}}\right)} \frac{d s}{s} \\
\quad \leq R^{\frac{d}{2}\left(\frac{1}{p}-\frac{1}{p^{\prime}}\right)} \int_{0}^{R} F(s) \frac{d s}{s} .
\end{aligned}
$$


We have proved that

$$
\|F(H)\|_{L^{p} \rightarrow L^{p^{\prime}}} \lesssim R^{\frac{d}{2}\left(\frac{1}{p}-\frac{1}{p^{\prime}}\right)} \int_{0}^{R} F(s) \frac{d s}{s} .
$$

Next, we extend the latter estimate to all bounded functions $F$ with support in $[0, R]$. This can be achieved by classical approximation arguments. First assume that the support of $F$ is contained in $[\eta, R]$ for some $\eta>0$ and apply (2.8) to the convolution $F_{\epsilon}=\rho_{\epsilon} * F$ by a mollifier $\rho_{\epsilon}$. We obtain

$$
\left\|F_{\epsilon}(H)\right\|_{L^{p} \rightarrow L^{p^{\prime}}} \lesssim(R+\epsilon)^{\frac{d}{2}\left(\frac{1}{p}-\frac{1}{p^{\prime}}\right)} \int_{\eta-\epsilon}^{R} F_{\epsilon}(s) \frac{d s}{s} .
$$

Since $\left|F_{\epsilon}(s)\right| \leq\|F\|_{\infty}$ and the support of $F_{\epsilon}$ is contained in $[\eta / 2, R+\eta / 2]$ for $\epsilon<\eta / 2$ one can apply the dominated convergence theorem to the RHS of the previous inequality. We obtain (2.8). Now for every bounded $F$ with support in $[0, R]$ we can apply (2.8) to $\chi_{[\epsilon, R]} F$ and then let $\epsilon \rightarrow 0$. Assertion (3) is then proved.

Finally we prove that (3) implies (1). In order to do this, we fix $\lambda>0$ and $\epsilon \in(0, \lambda)$, and apply (3) to $F(s)=\chi_{(\lambda-\epsilon, \lambda+\epsilon]}(s)$. It follows that

$$
\begin{aligned}
\left\|\chi_{(\lambda-\epsilon, \lambda+\epsilon]}(H)\right\|_{L^{p} \rightarrow L^{p^{\prime}}} & \lesssim(\lambda+\epsilon)^{\frac{d}{2}\left(\frac{1}{p}-\frac{1}{p^{\prime}}\right)} \int_{\lambda-\epsilon}^{\lambda+\epsilon} \frac{d s}{s} \\
& =(\lambda+\epsilon)^{\frac{d}{2}\left(\frac{1}{p}-\frac{1}{p^{\prime}}\right)}[\ln (\lambda+\epsilon)-\ln (\lambda-\epsilon)] \\
& \simeq(\lambda+\epsilon)^{\frac{d}{2}\left(\frac{1}{p}-\frac{1}{p^{\prime}}\right)} \frac{2 \epsilon}{\lambda}
\end{aligned}
$$

Hence,

$$
\left\|\epsilon^{-1} \chi_{(\lambda-\epsilon, \lambda+\epsilon]}(H)\right\|_{L^{p} \rightarrow L^{p^{\prime}}} \lesssim(\lambda+\epsilon)^{\frac{d}{2}\left(\frac{1}{p}-\frac{1}{p^{\prime}}\right)} \lambda^{-1}
$$

We let $\epsilon \rightarrow 0$ and obtain assertion (1).

\section{Remark 2.4.}

- In the proof of $(1) \Rightarrow(2)$ we can take $N=0$ and obtain that (1) implies

$$
\left\|e^{-t H}\right\|_{L^{p} \rightarrow L^{p^{\prime}}} \lesssim t^{-\frac{d}{2}\left(\frac{1}{p}-\frac{1}{p^{\prime}}\right)}, t>0 .
$$

- Suppose that (1.1) holds. Let $\alpha>0$ and apply assertion (3) with $F\left(s^{\alpha}\right)$ to obtain

$$
\left\|F\left(H^{\alpha}\right)\right\|_{L^{p} \rightarrow L^{p^{\prime}}} \lesssim R^{\frac{d}{2 \alpha}\left(\frac{1}{p}-\frac{1}{p^{\prime}}\right)} \int_{\mathbb{R}}\left|F\left(s^{\alpha}\right)\right| \frac{d s}{s}=\alpha R^{\frac{d}{2 \alpha}\left(\frac{1}{p}-\frac{1}{p^{\prime}}\right)} \int_{\mathbb{R}}|F(s)| \frac{d s}{s} .
$$

We conclude by Theorem 1.1 that

$$
\left\|\frac{d E_{H^{\alpha}}(\lambda)}{d \lambda}\right\|_{L^{p} \rightarrow L^{p^{\prime}}} \leq C \lambda^{\frac{d}{2 \alpha}\left(\frac{1}{p}-\frac{1}{p^{\prime}}\right)-1}, \quad \lambda>0
$$

In particular, for $\alpha=\frac{1}{2}$

$$
\left\|\frac{d E_{\sqrt{H}}(\lambda)}{d \lambda}\right\|_{L^{p \rightarrow L^{p^{\prime}}}} \leq C \lambda^{d\left(\frac{1}{p}-\frac{1}{p^{\prime}}\right)-1} .
$$


- Assume that the heat semigroup $\left(e^{-t H}\right)_{t>0}$ satisfies the classical $L^{p}-L^{2}$ estimates

$$
\left\|e^{-t H}\right\|_{L^{p} \rightarrow L^{2}} \lesssim t^{-\frac{d}{2}\left(\frac{1}{p}-\frac{1}{2}\right)} \quad \text { for every } t>0 \text { and some } p \in[1,2] .
$$

Then we observe that for every integer $N \geq 3$

$$
\begin{aligned}
\left\|H^{N} e^{-t H}\right\|_{L^{p} \rightarrow L^{p^{\prime}}} & \leq\left\|e^{-\frac{t}{N} H}\right\|_{L^{2} \rightarrow L^{p^{\prime}}}\left\|H^{N} e^{-t\left(1-\frac{2}{N}\right) H}\right\|_{L^{2} \rightarrow L^{2}}\left\|e^{-\frac{t}{N} H}\right\|_{L^{p} \rightarrow L^{2}} \\
& \lesssim\left(\frac{t}{N}\right)^{-\frac{d}{2}\left(\frac{1}{p}-\frac{1}{p^{\prime}}\right)}\left(\frac{N}{t\left(1-\frac{2}{N}\right)}\right)^{N} e^{-N} \\
& \lesssim t^{-N-\frac{d}{2}\left(\frac{1}{p}-\frac{1}{p^{\prime}}\right)} N^{\frac{d}{2}\left(\frac{1}{p}-\frac{1}{p^{\prime}}\right)}\left(N e^{-1}\right)^{N} \\
& \lesssim t^{-N-\frac{d}{2}\left(\frac{1}{p}-\frac{1}{p^{\prime}}\right)} N^{\frac{d}{2}\left(\frac{1}{p}-\frac{1}{p^{\prime}}\right)}(N-1) ! \sqrt{N}
\end{aligned}
$$

where we used Stirling's formula to obtain the last inequality. Therefore, we see that the gap between this very general estimate with the one required in Theorem 1.1 is an extra term of order $N^{\frac{1}{2}}$.

\section{Restriction from dispersion}

In this section we show that dispersive estimates for the semigroup generated by $H$ imply restriction estimates and also $L^{p}-L^{p^{\prime}}$ estimates for the derivatives $\frac{d^{k+1}}{d \lambda^{k+1}} E_{H}(\lambda)$ up to some order. The result for the case $k=0$ was already derived in [1] by a different proof. The result for $k \geq 1$ seems to be new.

Proposition 3.1. Fix $1 \leq p<\frac{2 d}{d+2}=2_{*}$. Suppose that the semigroup $\left(e^{-z H}\right)_{z \in \mathbb{C}^{+}}$ satisfies the following dispersive estimates:

$$
\left\|e^{-z H}\right\|_{L^{p} \rightarrow L^{p^{\prime}}} \lesssim|z|^{-\frac{d}{2}\left(\frac{1}{p}-\frac{1}{p^{\prime}}\right)},
$$

uniformly in $z$ such that $\Re(z)>0$. Then, for $\gamma>0$

$$
\left\|(N s H)^{N+\gamma} e^{-N s H}\right\|_{L^{p} \rightarrow L^{p^{\prime}}} \lesssim s^{-\frac{d}{2}\left(\frac{1}{p}-\frac{1}{p^{\prime}}\right)} N^{\gamma}(N-1) !
$$

uniformly in $N \geq 1$ and $s>0$ (the implicit constant only depends on $\gamma>0$ ).

Remark 3.2. It follows from Cauchy's formula that (3.1) extends to the derivatives of the semigroup as follows: for $k \geq 1$ and every $z \in \mathbb{C}^{+}$

$$
\left\|(\Re(z) H)^{k} e^{-z H}\right\|_{L^{p} \rightarrow L^{p^{\prime}}} \lesssim|z|^{-\frac{n}{2}\left(\frac{1}{p}-\frac{1}{p^{\prime}}\right)} .
$$

Proof. By the functional calculus, we have

$$
(N s H)^{N+\gamma} e^{-N s H}=N ! N^{N}(s N H)^{\gamma} \int_{\Gamma_{N}} \frac{e^{-\zeta s H}}{(\zeta-N)^{N+1}} d \zeta
$$


where $\Gamma_{N}$ is the circle of center $N$ and of radius $N-1$. So we deduce that

$$
\begin{aligned}
\left\|(N H s)^{N+\gamma} e^{-N s H}\right\|_{L^{p} \rightarrow L^{p^{\prime}}} & \leq N^{N+\gamma} N ! \int_{\Gamma_{N}} \frac{\left\|(s H)^{\gamma} e^{-\zeta s H}\right\|_{L^{p} \rightarrow L^{p^{\prime}}}}{|\zeta-N|^{N+1}} d \zeta \\
& \leq N^{N+\gamma} N !(N-1)^{-(N+1)} \int_{\Gamma_{N}}\left\|(s H)^{\gamma} e^{-\zeta s H}\right\|_{L^{p} \rightarrow L^{p^{\prime}}} d \zeta \\
& \lesssim N ! N^{\gamma} \int_{0}^{2 \pi}\left\|(s H)^{\gamma} e^{-\zeta_{\theta} s H}\right\|_{L^{p} \rightarrow L^{p^{\prime}}} d \theta
\end{aligned}
$$

with $\zeta_{\theta}:=N+(N-1) e^{i \theta}$. Writing (up to some numerical constant) with an integer $k \geq 1+\gamma$

$$
(s H)^{\gamma} e^{-\zeta s H}=s^{\gamma} \int_{0}^{\infty}(t H)^{k} e^{-(t+s \zeta) H} \frac{d t}{t^{1+\gamma}},
$$

we deduce that (since $\gamma \in(0, k))$

$$
\begin{aligned}
\left\|(s H)^{\gamma} e^{-\zeta s H}\right\|_{L^{p} \rightarrow L^{p^{\prime}}} & \lesssim s^{\gamma} \int_{0}^{\infty} \frac{t^{k}}{(t+s \Re(\zeta))^{k}(|t+s \zeta|)^{\frac{d}{2}\left(\frac{1}{p}-\frac{1}{p^{\prime}}\right)}} \frac{d t}{t^{1+\gamma}} \\
& \lesssim s^{\gamma}(s|\zeta|)^{-\frac{d}{2}\left(\frac{1}{p}-\frac{1}{p^{\prime}}\right)} \int_{0}^{\infty}\left(\frac{t}{t+s \Re(\zeta)}\right)^{k} \frac{d t}{t^{1+\gamma}} \\
& \lesssim(s|\zeta|)^{-\frac{d}{2}\left(\frac{1}{p}-\frac{1}{p^{\prime}}\right)} s^{\gamma}\left[\int_{0}^{s \Re(\zeta)}\left(\frac{t}{s \Re(\zeta)}\right)^{k} \frac{d t}{t^{1+\gamma}}+\int_{s \Re(\zeta)}^{\infty} \frac{d t}{t^{1+\gamma}}\right] \\
& \lesssim(s|\zeta|)^{-\frac{d}{2}\left(\frac{1}{p}-\frac{1}{p^{\prime}}\right)} \Re(\zeta)^{-\gamma} \\
& \lesssim(s|\zeta|)^{-\frac{d}{2}\left(\frac{1}{p}-\frac{1}{p^{\prime}}\right)},
\end{aligned}
$$

where we used that $\Re(\zeta) \geq 1$ and $|t+s \zeta| \geq|s \zeta|$. Putting this estimate together with (3.2) yields with $\sigma:=\frac{d}{2}\left(\frac{1}{p}-\frac{1}{p^{\prime}}\right)$

$$
\begin{aligned}
& \left\|(N s H)^{N+\gamma} e^{-N s H}\right\|_{L^{p} \rightarrow L^{p^{\prime}}} \\
& \lesssim s^{-\sigma} N ! N^{\gamma} \int_{0}^{2 \pi}\left|N+(N-1) e^{i \theta}\right|^{-\sigma} d \theta \\
& \lesssim s^{-\sigma} N ! N^{\gamma} \int_{-1}^{1}\left((N+(N-1) u)^{2}+(N-1)^{2}\left(1-u^{2}\right)\right)^{-\sigma / 2} \frac{d u}{\sqrt{1-u^{2}}} \\
& \lesssim s^{-\sigma} N ! N^{\gamma}\left[\int_{-1}^{0}\left(N^{2}(1+u)^{2}+(N-1)^{2}(1+u)\right)^{-\sigma / 2} \frac{d u}{\sqrt{1+u}}\right. \\
& \left.\quad+\int_{0}^{1} N^{-\sigma} \frac{d u}{\sqrt{1-u}}\right] \\
& \lesssim s^{-\sigma} N ! N^{\gamma}\left[\int_{0}^{(N-1)^{2}}(1+v)^{-\sigma / 2} \frac{d v}{N \sqrt{v}}+N^{-\sigma}\right] \\
& \lesssim s^{-\sigma} N ! N^{\gamma-1} \simeq s^{-\sigma}(N-1) ! N^{\gamma},
\end{aligned}
$$

where we used that $\sigma>1$ since $p<2$. 
Corollary 3.3. Assume that the semigroup $\left(e^{-z H}\right)_{z \in \mathbb{C}^{+}}$satisfies the dispersive estimate (3.1) for some $1 \leq p<\frac{2 d}{d+2}=2_{*}$. Then we have

$$
\left\|\frac{d E_{H}(\lambda)}{d \lambda}\right\|_{L^{p} \rightarrow L^{p^{\prime}}} \lesssim \lambda^{\frac{d}{2}\left(\frac{1}{p}-\frac{1}{p^{\prime}}\right)-1}, \lambda>0 .
$$

In addition for an integer $k \leq d / 2-1$, if $1 \leq p<\frac{2 d}{d+2(k+1)}$ then

$$
\left\|\frac{d^{k+1}}{d \lambda^{k+1}} E_{H}(\lambda)\right\|_{L^{p} \rightarrow L^{p^{\prime}}} \lesssim \lambda^{\frac{d}{2}\left(\frac{1}{p}-\frac{1}{p^{\prime}}\right)-(k+1)}, \lambda>0 .
$$

Proof. The first assertion follows immediately from Proposition 3.1 and Theorem 1.1. For the second assertion we give for simplicity a proof for $k=1$, the general case follows by iteration. By Corollary 2.3 we have in the weak sense

$$
\begin{aligned}
\frac{d^{2}}{d \lambda^{2}} E_{H}(\lambda) & =\lim _{N \rightarrow \infty} \frac{1}{N !} \frac{d}{d \lambda}\left[\left(N \lambda^{-1} H\right)^{N+1} e^{-\lambda^{-1} N H} \lambda^{-1}\right] \\
& =\lim _{N \rightarrow \infty} \frac{1}{N !}(N H)^{-1} \frac{d}{d \lambda}\left[\left(N \lambda^{-1} H\right)^{N+2} e^{-\lambda^{-1} N H}\right] .
\end{aligned}
$$

Following Proposition 3.1, we write

$$
\left(N \lambda^{-1} H\right)^{N+2} e^{-N \lambda^{-1} H}=(N+2) ! N^{N+2} \int_{\Gamma_{N}} \frac{e^{-\zeta \lambda^{-1} H}}{(\zeta-N)^{N+3}} d \zeta
$$

and so

$$
\frac{d}{d \lambda}\left[\left(N \lambda^{-1} H\right)^{N+2} e^{-N \lambda^{-1} H}\right]=(N+2) ! N^{N+2} \lambda^{-2} \int_{\Gamma_{N}} \frac{\zeta H e^{-\zeta \lambda^{-1} H}}{(\zeta-N)^{N+1}} d \zeta .
$$

Hence,

$$
\frac{1}{N !}(N H)^{-1} \frac{d}{d \lambda}\left[\left(N \lambda^{-1} H\right)^{N+2} e^{-\lambda^{-1} N H}\right]=\frac{(N+2) !}{(N-1) !} N^{N} \lambda^{-2} \int_{\Gamma_{N}} \frac{\zeta e^{-\zeta \lambda^{-1} H}}{(\zeta-N)^{N+1}} d \zeta .
$$

Using the dispersive estimate, it follows that

$$
\begin{aligned}
\frac{1}{N !} & (N H)^{-1}\left\|\frac{d}{d \lambda}\left[\left(N \lambda^{-1} H\right)^{N+2} e^{-\lambda^{-1} N H}\right]\right\|_{L^{p} \rightarrow L^{p^{\prime}}} \\
& \lesssim \frac{(N+2) !}{(N-1) !} N^{N} \lambda^{-2} \int_{\Gamma_{N}} \frac{|\zeta|\left\|e^{-\zeta \lambda^{-1} H}\right\|_{L^{p} \rightarrow L^{p^{\prime}}}}{|\zeta-N|^{N+1}} d \zeta \\
& \lesssim \frac{(N+2) !}{(N-1) !} \frac{N^{N}}{(N-1)^{N+1}} \lambda^{-2} \int_{\Gamma_{N}}|\zeta|^{1-\sigma} \lambda^{\sigma} d \zeta
\end{aligned}
$$

with $\sigma:=\frac{d}{2}\left(\frac{1}{p}-\frac{1}{p^{\prime}}\right)$. Such integral was already computed in Proposition 3.1 and is uniformly bounded as soon as $1-\sigma<-1$. This gives the desired estimate for $\frac{d^{2}}{d \lambda^{2}} E_{H}(\lambda)$.

\section{Acknowledgments}

Both authors are partly supported by the ANR under the project "Harmonic Analysis at its Boundaries" no. ANR-12-BS01-0013. The first author is also partly supported by the ANR under the project AFoMEN no. 2011-JS01-001-01. 


\section{References}

[1] P. Chen, E.M. Ouhabaz, A. Sikora and L. Yan, Restriction estimates, sharp spectral multipliers and endpoint estimates for Bochner-Riesz means, Submitted 2011, http://arxiv. org/abs/1202.4052.

[2] L. Grafakos, Classical Fourier Analysis, Graduate Texts in Mathematics, 2nd ed., 249, Springer, New York, NY, 2009.

[3] C. Guillarmou, A. Hassel and A. Sikora, Restriction and spectral multiplier theorems on asymptotically conic manifolds, Anal. PDE 6(4) (2013), 893-950.

CNRS-Université de Nantes, Laboratoire Jean Leray, 2, Rue De la Houssinière, 44322 NANTES CEDEX 3, FRANCE

E-mail address: Frederic.Bernicot@univ-nantes.fr

Institut de Mathématiques (IMB), Univ. Bordeaux 351, Cours de la LibÉration, 33405 TALENCE CEDEX, FRANCE

E-mail address: Elmaati.Ouhabaz@math.u-bordeaux1.fr 